Research Paper

\title{
Exosomes Derived from Human Endothelial Progenitor Cells Accelerate Cutaneous Wound Healing by Promoting Angiogenesis Through Erk1/2 Signaling
}

\author{
Jieyuan Zhang 1,2, ${ }^{*}$, Chunyuan Chen 1,3, ${ }^{*}$, Bin Hu ${ }^{1}$, Xin Niu ${ }^{1}$, Xiaolin Liu 1,2, Guowei Zhang 1, Changqing \\ Zhang ${ }^{1,2}$, Qing Li ${ }^{1 凶}$, Yang Wang ${ }^{1 凶}$ \\ 1. Institute of Microsurgery on Extremities, Shanghai Jiao Tong University Affiliated Sixth People's Hospital, 600 Yishan Road, Shanghai 200233, China; \\ 2. Department of Orthopedic Surgery, Shanghai Jiao Tong University Affiliated Sixth People's Hospital, 600 Yishan Road, Shanghai 200233, China; \\ 3. Graduate School of Nanchang University, 461 Bayi Road, Nanchang 330006, China. \\ * These authors contributed equally to this work. \\ $\square$ Corresponding authors: Yang Wang, Institute of Microsurgery on Extremities, Shanghai Jiao Tong University Affiliated Sixth People's Hospital, Shanghai \\ 200233, China. Tel.: +86-21-24056319; Fax: +86-21-64363802; E-mail: wangyang63@sjtu.edu.cn Co-correspondence: Qing Li, Institute of Microsurgery on \\ Extremities, Shanghai Jiao Tong University Affiliated Sixth People's Hospital, Shanghai 200233, China. E-mail: liqing_236@aliyun.com.
}

() Ivyspring International Publisher. Reproduction is permitted for personal, noncommercial use, provided that the article is in whole, unmodified, and properly cited. See http://ivyspring.com/terms for terms and conditions.

Received: 2016.03.11; Accepted: 2016.10.09; Published: 2016.11.25

\begin{abstract}
Chronic skin wounds represent one of the most common and disabling complications of diabetes. Endothelial progenitor cells (EPCs) are precursors of endothelial cells and can enhance diabetic wound repair by facilitating neovascularization. Recent studies indicate that the transplanted cells exert therapeutic effects primarily via a paracrine mechanism and exosomes are an important paracrine factor that can be directly used as therapeutic agents for regenerative medicine. However, application of exosomes in diabetic wound repair has been rarely reported. In this study, we demonstrated that the exosomes derived from human umbilical cord blood-derived EPCs (EPC-Exos) possessed robust pro-angiogenic and wound healing effects in streptozotocin-induced diabetic rats. By using a series of in vitro functional assays, we found that EPC-Exos could be incorporated into endothelial cells and significantly enhance endothelial cells' proliferation, migration, and angiogenic tubule formation. Moreover, microarray analyses indicated that exosomes treatment markedly altered the expression of a class of genes involved in Erk1/2 signaling pathway. It was further confirmed with functional study that this signaling process was the critical mediator during the exosomes-induced angiogenic responses of endothelial cells. Therefore, EPC-Exos are able to stimulate angiogenic activities of endothelial cells by activating Erk1/2 signaling, which finally facilitates cutaneous wound repair and regeneration.
\end{abstract}

Key words: Exosomes, Angiogenesis, Wound healing, Microarray, Erk1/2.

\section{Introduction}

The incidence of diabetes mellitus has increased world-wide and the complications arising from the disease have become serious public health problem [1]. Impaired skin wound healing is one of the most common and disabling complications of diabetes, which could diminish physical activity and lead to chronic ischemic skin lesions and even limb amputation [2]. Angiogenesis, the sprouting of capillaries from pre-existing blood vessels, is thought to be important for wound healing, as it plays a crucial role in delivery of oxygen and nutrients to the wound sites for sustaining fibroblast proliferation, collagen synthesis, and re-epithelialization [3]. However, angiogenesis usually gets compromised in patients with diabetes, which consequently lead to delayed wound healing [4]. Thus, strategies designed to augment the local angiogenesis responses may dramatically accelerate the diabetic wound healing process.

Over the past few years, cell-based therapy has emerged as a promising therapeutic approach for diabetic wound repair and regeneration. 
Accumulating studies showed that the transplantation of endothelial progenitor cells (EPCs) derived from human peripheral blood or umbilical cord blood (UCB) can stimulate new capillaries formation and wound closure in diabetic animal models $[5,6]$. EPCs are the precursors of endothelial cells and can differentiate into mature endothelial cells that directly contribute to angiogenesis and vascular regeneration [7]. Moreover, EPCs are able to secrete potent combinations of trophic factors that evoke angiogenic and regenerative responses of recipient cells [7]. These features make EPCs an attractive cell source for the treatment of chronic non-healing diabetic wounds. However, the direct use of stem/progenitor cells remains limited by issues of potential immunological rejection, chromosomal variation, emboli formation, etc [8-10]. Hence, it is imperative to develop a novel strategy that can fully exert the therapeutic effects of stem/progenitor cells and avoid the risks associated with the direct use of them.

In recent years, numerous studies have indicated that the activation of tissue-resident endothelial cells via paracrine mechanisms may be more crucial for EPCs-mediated neovascularization than their direct differentiation and incorporation into vasculature [5, 11]. Exosomes, an important component of cell paracrine secretion, are 40-150 nm-sized nanoparticles originating from multivesicular bodies (MVBs) [12]. They play crucial roles in intercellular communication by transferring genetic materials and proteins to target cells and thereby altering the gene and protein levels of the recipient cells to regulate their function [12]. It has been reported that exosomes exhibit similar functional properties to the cells from which they are derived and have no apparent adverse effects such as immunogenicity, malignant transformation, and vascular obstructive risks, suggesting that the exosomes-based therapy is much safer and promising for tissue regenerative treatments than the direct use of cells [13-15].

In the present study, we used the human UCB-derived EPCs as a "factory" to generate exosomes and explored whether these exosomes could accelerate angiogenesis and wound healing in STZ-induced diabetic rat models. The results demonstrated that the local injection of EPC-Exos into the skin wounds of diabetic rats markedly enhanced the wound healing process and the formation of new blood vessels in the wound sites. Since endothelial cells play a key role in angiogenesis [16, 17], we evaluated the effects of these exosomes on the angiogenic activities of human microvascular endothelial cells (HMECs) in vitro. The results showed that EPC-Exos could be internalized by
HMECs and remarkably enhance HMECs' proliferation, migration, and angiogenic tubule formation. Then, by gene expression profiling analyses, we found that exosomes treatment markedly altered the expression of a class of genes involved in extracellular signal-regulated kinases 1 and $2($ Erk1/2) signaling pathway. We used a specific inhibitor (U0126) to interfere with Erk1/2 signaling and found that inhibition of this signaling process profoundly reduced the pro-angiogenic effects by EPC-Exos. The data suggest that EPC-Exos can stimulate angiogenesis by activating Erk1/2 signaling, which finally facilitates wound repair and regeneration. To the best of our knowledge, this study is the first to show the therapeutic potential of EPC-Exos in diabetic wound healing and elucidate the underlying mechanism.

\section{Materials and methods}

\section{Isolation and culture of EPCs derived from human UCB}

The human UCB samples were obtained from the healthy newborns with permission from the infants' parents and the Institutional Review Board at Shanghai Six People's Hospital. Briefly, UCB diluted 1:1 in phosphate buffered saline (PBS) was overlaid onto the separation medium (Cappel LSM; MP Biomedicals, USA) and centrifuged at $400 \times \mathrm{g}$ for 30 minutes (min) at room temperature. The mononuclear cells (MNCs) layer were collected and washed with PBS. MNCs $\left(5 \times 10^{6}\right.$ cells $\left./ \mathrm{cm}^{2}\right)$ were then placed into 6-well plates precoated with type I rat tail collagen (BD Bioscience, Bedford, USA) and incubated in endothelial basal medium 2 (EBM-2; Lonza, USA) supplemented with EGM-2 MV SingleQuots containing 5\% FBS and cytokines. Cells were cultured at $37{ }^{\circ} \mathrm{C}, 5 \% \mathrm{CO}_{2}$ in a humidified environment. The media was changed every other day until the first passage. EPC colonies were enumerated by an inverted microscope (Leica BME, Germany). Cells were passaged at $80-90 \%$ confluence. Early-passage EPCs (p2-6) were used in the experiments as described below.

\section{Characterization of human UCB-derived EPCs}

\section{Immunocytochemistry}

Cells were fixed with $4 \%$ paraformaldehyde for 15 min and permeabilized with $0.1 \%$ Triton X-100 for $10 \mathrm{~min}$ at room temperature. After blocking with 3\% BSA for 1 hour, cells were incubated with primary antibodies overnight at $4{ }^{\circ} \mathrm{C}$ and then incubated with secondary antibodies for 1 hour at $37^{\circ} \mathrm{C}$. Irrelevant isotype-matched antibodies were used as negative controls. Nuclei were stained by 
4,6-diamidino-2-phenylindole (DAPI; $\quad 0.5 \mu \mathrm{g} / \mathrm{ml}$; Invitrogen, USA) for $5 \mathrm{~min}$. Cells were washed and analyzed using a fluorescence microscope (Leica DMI6000B, Germany). The antibodies including anti-von Willebrands Factor (vWF), anti-CD31, anti-CD34, and respective secondary antibodies were obtained from Abcam (Cambridge, Cambs, Britain).

\section{Flow cytometry analysis}

$5 \times 10^{5}$ cells were blocked with $3 \%$ BSA for 30 min and incubated with the following primary antibodies according to the manufacturer's instructions: FITC-conjugated anti-CD31, anti-CD45, and anti-vascular endothelial cadherin (VE-cadherin); PE-conjugated anti-CD133 and anti-vascular endothelial growth factor receptor-2 (VEGFR-2); APC-conjugated anti-CD34. Nonspecific fluorescence was determined by incubation of similar cell aliquots with isotype-matched mouse monoclonal antibodies. Cells were then washed and analyzed using the Guava easyCyte ${ }^{\mathrm{TM}}$ Flow Cytometer (Millipore, Billerica, USA). All antibodies were purchased from BD Biosciences (San Jose, CA, USA).

\section{Tube formation assay}

In vitro capillary-like structure formation was evaluated on growth factor reduced Matrigel (BD Biosciences, Bedford, USA). Briefly, Matrigel (50 $\mu \mathrm{L}$ per well) was added to the flat-bottomed 96-well plates and incubated at $37^{\circ} \mathrm{C}$ for $30 \mathrm{~min}$. Cells $\left(2 \times 10^{4}\right.$ cells per well) were seeded on Matrigel and cultured for 8 hours. Tube formation was assessed using an inverted microscope (Leica DMI6000B, Germany).

\section{Assessment of acetylated low density lipoprotein (ac-LDL) uptake and ulex europaeus agglutinin-1 (UEA-I) binding}

The uptake of DiL-labeled ac-LDL (Dil-ac-LDL; Molecular Probes, Eugene, USA) and binding of FITC-conjugated UEA-1 (FITC-UEA-1; Sigma-Aldrich, USA) by EPCs were assessed by fluorescent staining. Briefly, cells were incubated with Dil-ac-LDL (15 $\mu \mathrm{g} / \mathrm{mL}$ ) for 4 hours at $37{ }^{\circ} \mathrm{C}$ and fixed with $4 \%$ paraformaldehyde for $30 \mathrm{~min}$. After washing, cells were stained with FITC-UEA-l $(10 \mu \mathrm{g} / \mathrm{mL})$ for 1 hour at $37{ }^{\circ} \mathrm{C}$ and with DAPI for $5 \mathrm{~min}$. Cells were washed and analyzed using a fluorescence microscope (Leica DMI6000B, Germany).

\section{Isolation and identification of human UCB-derived EPCs-Exos}

After EPCs becoming 80-90\% confluent, EPCs were rinsed with PBS and cultured in EGM-2MV media deprived of FBS and supplemented with $1 \times$ serum replacement solution (PeproTech, USA) for an additional 24 hours. The conditioned media of EPCs was obtained and centrifuged at $300 \times \mathrm{g}$ for $10 \mathrm{~min}$ and $2000 \times \mathrm{g}$ for $10 \mathrm{~min}$ to remove dead cells and cellular debris. Then, the supernatant was filtered using a $0.22 \mu \mathrm{m}$ filter (Millipore) and centrifuged at $4000 \times \mathrm{g}$ to about $200 \mu \mathrm{L}$ by ultra-filtration in a $15 \mathrm{~mL}$ Amicon Ultra-15 Centrifugal Filter Unit (Millipore, Billerica MA, USA). The ultrafiltration liquid was washed twice with PBS and re-ultrafiltrated at $4000 \times$ $\mathrm{g}$ to $200 \mu \mathrm{L}$. For exosomes purification, the liquid was overlaid onto $30 \%$ sucrose- $\mathrm{D}_{2} \mathrm{O}$ cushion in a sterile Ultra-Clear ${ }^{\mathrm{TM}}$ tube (Beckman Coulter, Kraemer Boulevard Brea, USA) and ultracentrifuged at 100,000 $\times \mathrm{g}$ for one hour. The pelleted exosomes were resuspended in $15 \mathrm{~mL}$ of PBS and centrifuged at 4000 $\times \mathrm{g}$ to about $200 \mu \mathrm{L}$ in Centrifugal Filter Units. All procedures were performed at $4{ }^{\circ} \mathrm{C}$. Exosomes were stored at $-80{ }^{\circ} \mathrm{C}$ or used for downstream experiments.

Tunable resistive pulse sensing (TRPS) analysis, transmission electron microscopy (TEM), and western blotting were used to identify the collected exosomes. The size distribution and concentration of exosomes were measured by TRPS analysis using a qNano platform with an NP100-rated nanopore (Izon Science, UK). The membrane was stretched at 43.0 $\mathrm{mm}$. All measurements were calibrated with $70 \mathrm{~nm}$ (CPC100) polystyrene beads (Izon Science, UK). Samples were diluted 1000-fold with $0.22-\mu \mathrm{m}$ filtered PBS and measured for three times. Data processing and analysis were carried out on the Izon Control Suite software v2.2 (Izon Science, UK). The morphologies of exosomes were observed by TEM. Briefly, exosomes were fixed in 3\% glutaraldehyde for 2 hours, washed with PBS, and then negatively stained with 2\% uranyl acetate for 30 seconds. Exosomes were then loaded on a continuous carbon grid and visualized by a Hitachi H-7650 transmission electron microscope (Hitachi, Tokyo, Japan). The exosomal characteristic surface marker proteins including CD9, CD63, and CD81 and the endothelial lineage marker CD31 were analyzed by western blotting.

\section{Diabetic skin wound animal model and treatment}

All procedures were approved by the Animal Research Committee of Shanghai Jiao Tong University Affiliated Sixth People's Hospital. Adult male sprague dawley (SD) rats weighing 250-300 g were used in this study. Experimental diabetes mellitus was induced by a single intraperitoneal injection of STZ (Sigma, St. Louis, MO, USA; $60 \mathrm{mg} / \mathrm{kg}$ ) [18]. Blood glucose levels were monitored after 72 hours and those rats showing blood glucose level above $300 \mathrm{mg} / \mathrm{dl}$ were selected for further study. The diabetic rats were kept 
under observation for 14 days before creation of skin wounds.

Ten STZ-induced diabetic rats were anesthetized by intraperitoneal injection of $50 \mathrm{mg} / \mathrm{kg}$ pentobarbital. After shaving the rats, three $15 \mathrm{~mm}$ diameter full-thickness excisional wounds were created on the upper back. Wounds were randomly assigned to three treatment groups, which were subcutaneously injected with exosomes $\left(2 \times 10^{10}\right.$ or $1 \times$ $10^{11}$ particles, dissolved in $200 \mu \mathrm{L}$ of PBS) or an equal volume of PBS around the wounds at 4 injection sites. Six wounds per group were studied by histopathological analysis at day 14 post-wounding. Wound-size reduction was calculated using the equation: wound-size reduction $(\%)=\left(\mathrm{A}_{\mathrm{O}}-\right.$ $\left.A_{t}\right) / A_{O} \times 100$, where $A_{O}$ is the initial wound area, and $A_{t}$ is the wound area at day 4,7 or 14 post-wounding.

\section{Microfil perfusion and micro-computed tomography (micro-CT)}

To compare blood vessels formation in the defects areas, four rats were perfused with Microfil (Microfil MV-122; Flow Tech, USA) at day 14 post-wounding. Briefly, all of the rats were anesthetized for rib cage opening. The descending aorta was clamped, and an angiocatheter was used to penetrate the left ventricle. After the inferior vena cava was incised, heparinized saline was perfused until the venous effluent was free of blood, and $20 \mathrm{~mL}$ of Microfil was perfused at $2 \mathrm{~mL} / \mathrm{min}$. The rats were then maintained overnight at $4{ }^{\circ} \mathrm{C}$ to ensure polymerization of the contrast agent. The defects were scanned using micro-CT (Skyscan 1176; Kontich, Belgium) at 9- $\mu \mathrm{m}$ resolution to detect the new blood vessels. 3D images were reconstructed by using the auxiliary software, and the blood vessels areas in the defects were calculated.

\section{Histological and immunofluorescence analysis}

For histological analysis, the excised tissues composed of wound bed and surrounding healthy skins at day 14 post-wounding were fixed in 10\% formalin, dehydrated with a graded-alcohol series, embedded in paraffin, and sectioned perpendicularly to the wound surface into 4 - $\mu$ m-thick sections. Hematoxylin and eosin (H\&E) staining was used for histological observations. The percentage of re-epithelialization $(\mathrm{E} \%)$ was calculated using the equation: $E \%=W_{N} / W_{O} \times 100$, where $W_{O}$ is the original wound area, and $W_{N}$ is the length of newly generated epithelium across the surface of the wound. Masson's trichrome staining was adopted to determine the degree of collagen maturity in the wound beds.

Immunofluorescence staining for CD31 (mouse anti-rat) and alpha smooth muscle actin (a-SMA, rabbit anti-rat) was done to determine the extent of blood vessels formation in granulation tissues at day 14 post-wounding. ki67 (rabbit anti-rat) and CD31 (mouse andti-rat) co-staining was done to evaluate the effect of EPC-Exos on the proliferation of endothelial cells in the wound sites. Briefly, excised tissues at day 14 post-wounding were fixed in $4 \%$ paraformaldehyde, dehydrated in $30 \%$ sucrose solution, embedded in OCT, and sectioned perpendicularly to the wound surface into 4- $\mu \mathrm{m}$-thick sections. Tissue sections were blocked in 1\% BSA for $30 \mathrm{~min}$ at room temperature, incubated with primary antibody overnight at $4{ }^{\circ} \mathrm{C}$, and then incubated with respective secondary antibody (goat anti-rabbit or goat anti-mouse) for one hour at room temperature. All antibodies were obtained from Abcam (Cambridge, Cambs, Britain). Images were acquired with a fluorescence microscope (IX81; Olympus, Hamburg, Germany). The numbers of blood vessels in different groups were determined by counting in six random fields per section between wound edges using Image-Pro Plus 6.

\section{In vitro effects of EPC-Exos on vascular endothelial cells}

\section{Cells culture}

Cells from human microvascular endothelial cell line (HMEC-1) [19] (Centers for Disease Control and Prevention, Atlanta, GA) were cultured in MCDB131 cell culture media (Gibco BRL, Grand Island, USA) containing 10\% FBS (Gibco BRL), 2 mM L-glutamine (Sigma, St. Louis, MO), $10 \mathrm{ng} / \mathrm{mL}$ epidermal growth factor (Sigma), and $1 \mu \mathrm{g} / \mathrm{mL}$ hydrocortisone (Sigma). HMECs were maintained at $37{ }^{\circ} \mathrm{C}, 5 \% \mathrm{CO}_{2}$.

\section{Exosomes uptake by endothelial cells}

EPCs were labeled with Vybrant $\mathrm{DiO}$ dye (Molecular Probes, Carlsbad, CA, USA) according to the manufacturer's instructions. Briefly, cells were trypsinized and resuspended in $1 \mathrm{~mL}$ of serum-free EGM-2MV media. $5 \mu \mathrm{L}$ of the cell-labeling solution was added to the cells, followed by incubation at 37 ${ }^{\circ} \mathrm{C}, 5 \% \mathrm{CO}_{2}$ for $15 \mathrm{~min}$. The cell-labeled suspension was centrifuged at $300 \times \mathrm{g}$ for $15 \mathrm{~min}$ and the supernatant was discarded. Cells were washed with PBS and cultured for an additional 24 hours. Subsequently, the exosomes were isolated and purified from the EPCs-derived culture medium, and then incubated with HMECs at $37{ }^{\circ} \mathrm{C}$ for 2 hours. HMECs were washed with PBS, fixed with $4 \%$ paraformaldehyde for $15 \mathrm{~min}$, and stained with DAPI for $5 \mathrm{~min}$ at room temperature. After washing, cells were analyzed with a fluorescence microscope (Leica 
DMI6000B, Solms, Germany).

\section{Cells proliferation assay}

A Cell Counting Kit-8 assay (CCK-8; Dojindo, Kyushu Island, Japan) was used to assess cell proliferation. HMECs $\left(5 \times 10^{3}\right.$ cells per well $)$ were seeded onto 96-well plates and cultured in serum-free MCDB131 media containing $2 \times 10^{10}$ or $1 \times 10^{11}$ particles $/ \mathrm{mL}$ of exosomes or an equal volume of exosome diluent (PBS). A group without cells served as the blank. At day 1, 2, 3, 4, and 5, CCK-8 solution (10 $\mu \mathrm{L}$ per well) was added to HMECs and cells were incubated at $37^{\circ} \mathrm{C}$ for 3 hours. The absorbance was measured at $450 \mathrm{~nm}$ by using a microplate reader and the optical density values represented the survival/proliferation of HMECs.

\section{Tube formation assay}

HMECs $\left(2 \times 10^{4}\right.$ cells per well $)$ were seeded onto Matrigel-coated 96-well plates and incubated in serum-free MCDB131 media containing exosomes $(2 \times$ $10^{10}$ or $1 \times 10^{11}$ particles $/ \mathrm{mL}$ ) or PBS at $37^{\circ} \mathrm{C}$. Tube formation was examined 4 hours $(t=4 \mathrm{hr}$ ) and 8 hours $(\mathrm{t}=8 \mathrm{hr}$ ) later by an inverted microscope (Leica DMI6000B, Germany). The total branching points, total tube length, cell covered area, and total loops per image were measured by a blinded independent observer.

\section{Migration assay}

HMECs $\left(2 \times 10^{5}\right.$ cells per well $)$ were plated in 12-well plates and incubated at $37{ }^{\circ} \mathrm{C}$. After cells had attached, the confluent monolayer was scratched using a p200 pipette tip and washed with PBS to remove the debris and smooth the edge of the scratch. $1 \mathrm{~mL}$ of serum-free MCDB131 media containing exosomes $\left(2 \times 10^{10}\right.$ or $1 \times 10^{11}$ particles $\left./ \mathrm{mL}\right)$ or PBS was added. Cells were photographed immediately $(\mathrm{t}=0 \mathrm{hr}), 8$ hours $(\mathrm{t}=8 \mathrm{hr})$ and 12 hours $(\mathrm{t}=12 \mathrm{hr})$ later. The level of migration area was assessed by the ratio of closure area to initial wound $(\mathrm{t}=0 \mathrm{hr})$ as follows: migration area $(\%)=(A 0-A n) / A 0 \times 100$, where $A 0$ represents the area of initial wound area, $A n$ represents the residual area of wound at the metering point $(\mathrm{t}=\mathrm{n} \mathrm{hr})$.

\section{Gene expression profiling analysis}

HMECs $\left(1 \times 10^{6}\right.$ cells per well $)$ were seeded in $25-\mathrm{cm}^{2}$ culture flask and incubated in serum-free MCDB131 media supplemented with exosomes $(1 \times$ $10^{11}$ particles $/ \mathrm{mL}$ ) or PBS at $37^{\circ} \mathrm{C}$ for 24 hours. Total RNA from HMECs was extracted using the TRIZOL Reagent (Invitrogen, Carlsbad, CA, USA). Gene expression analyses on Affymetrix GeneChip PrimeView Human Gene Expression Array were performed by Shanghai Biotechnology Corporation. The experiments were performed in triplicates. The data were analyzed using Genespring Software 11.0 (Agilent Technologies). The labeling and hybridization were performed according to the protocols in the Affymetrix GeneChip 3' IVT Express Kit User Manual. The slides were scanned by GeneChip ${ }^{\circledR}$ Scanner 3000. Raw data were normalized by MAS5.0s algorithm and expressed in log2 scale. The differentially expressed genes (DEGs) were identified with cutoff of absolute fold change $\geq 1.5$ and $p$ value $<0.05$ by student's $t$ test. Then, the DEGs were biologically interpreted using the Gene ontology (GO) database (geneontology.org) and Kyoto Encyclopedia of Genes and Genomes (KEGG) public pathway resource (www.genome.jp/kegg). The DEGs were then illustrated as a heat map by using $\mathrm{R}$ package (www.r-project.org). All microarray data are available in the Gene Expression Omnibus under accession no. GSE77048.

\section{Quantitative real-time PCR Analysis (qRT-PCR)}

HMECs $\left(5 \times 10^{5}\right.$ cells per well $)$ were seeded in 6-well plates and cultured in serum- free MCDB131 media supplemented with exosomes $\left(1 \times 10^{11}\right.$ particles/mL) or PBS for 24 hours. Total RNA was extracted using TRIzol Reagent (Invitrogen, Carlsbad, USA) and cDNA was synthesized from $1 \mu \mathrm{g}$ of total RNA by using the Revert Aid first-strand cDNA synthesis kit (Fermentas, Life Sciences, Canada). Then, qRT-PCR was performed with an ABI PRISM®7900HT System with SYBR Premix ExTaq ${ }^{\mathrm{TM}}$ II (Takara Biotechnology, Japan). GAPDH was used as a housekeeping gene for internal normalization. Primers used in the amplification reaction are synthesized as followed: FGF2: forward, 5'-CAATTCCCATGTGCTGTGAC-3', and reverse, 5'-ACCTTGACCTCTCAGCCTCA-3'; IL6: forward, $5^{\prime}$-AGTTCCTGCAGAAAAAGGCAAAG-3', and reverse, 5'-ACCTTGACCT CTCAGCCTCA-3'; IL8: forward, 5'-ACCGGAAGGAACCATCTCAC-3', and reverse, 5'-GGCAAAACTGCACCTTCACAC-3'; c-MYC: forward, 5'-TTACAACACCCGAGCAA GGA- $3^{\prime}$, and reverse, $5^{\prime}$-AAATACGGCTGCACCGA GTC-3'; ID1: forward, 5'-GGAATCCGAAGTTGGAA CCC- 3 ', and reverse, $5^{\prime}$-GGCAAAACTGCACCTTCA CAC-3'; COX2: forward, 5'-GATGATTGCCCGACTC CCTT-3', and reverse, $5^{\prime}$-GAAAAGGCGCAGTTTAC GCT-3'; VEGFA: forward, 5'-ATCACCATGCAGATT ATGCGG-3' ${ }^{\prime}$, and reverse, $5^{\prime}$-CCCCTTTCCCTTTCCT CGAAC-3'; CCND1: forward, 5'-CCTGTGCTGCGAA GTGGAAAC- $3^{\prime}$, and reverse, $5^{\prime}$-TCGCACTTCTGTTC CTCGCA-3'; GAPDH: forward, 5'-ATCCCATCACC ATCTTCC-3', and reverse, 5'-GAGTCCTTCCACGAT 
ACCA - $3^{\prime}$.

\section{Western blotting analysis}

Cells or purified exosomes samples were diluted 1:5 with protein loading buffer $(6 \times)$ (Transgen Biotech, Beijing, China) and heated at $95^{\circ} \mathrm{C}$ for $5 \mathrm{~min}$. Protein extracts were separated on a $10 \%$ sodium dodecyl sulfate-polyacrylamide gel electrophoresis (SDS-PAGE) gel, blotted onto polyvinylidene fluoride membranes (Whatman, Maidstone, Kent, Britain), and then blocked with 5\% non-fat dried milk in TBST (10 $\mathrm{mM}$ Tris- $\mathrm{HCl} \mathrm{pH}$ 7.5, $150 \mathrm{mM} \mathrm{NaCl}, 0.1 \%$ Tween-20). The membranes were incubated with primary antibodies at $4{ }^{\circ} \mathrm{C}$ overnight, followed by incubation with the horseradish peroxidase (HRP)-conjugated secondary antibodies at $37{ }^{\circ} \mathrm{C}$ for 1 hour. The antibodies including anti-CD9, anti-CD63, anti-CD81, anti-CD31, anti-VEGFA, and anti-inhibitor of DNA binding 1 (Id1) were obtained from Abcam (Cambridge, Cambs, Britain), and anti-cyclooxygenase-2 (Cox-2), anti-c-Myc, anti-phosphorylate Erk1/2 (p-Erk1/2), anti-Erk1/2, anti-beta-actin, and all secondary antibodies were obtained from Cell Signaling Technology (Danvers, MA, USA). The immunoreactive bands were visualized using enhanced chemiluminescence reagent (Thermo Fisher Scientific, Waltham, USA) and imaged by an FluorChem HD2 gel documentation system (Proteinsimple, Santa Clara, CA).

\section{ERK1/2 signaling inhibition}

U0126 (1,4-diamino-2,3-dicyano-1,4-bis[2aminophenylthio] butadiene), a highly potent and selective inhibitor of MEK1/2 to block the Erk1/2 signaling activation, was purchased from Cell Signaling Technology (Danvers, MA, USA) and dissolved in dimethylsulfoxide (DMSO) at a stock concentration of $10 \mathrm{mM}$. To confirm the involvement of Erk1/2 signaling in exosomes-mediated effects on HMECs, cells were pre-treated with the indicated concentration $(10 \mu \mathrm{M})$ of $\mathrm{U} 0126$ or an equal volume DMSO for 2 hours. Next, $1 \times 10^{11}$ particles $/ \mathrm{mL}$ of exosomes or an equal volume of PBS was added to the culture medium of HMECs and the cells were cultured for 24 hours. Then, qRT-PCR, western blotting, and a series of angiogenesis-related assays on HMECs were then performed as above-described.

\section{Statistical analysis}

All of these experiments were performed at least three times. The data are shown as means \pm standard deviation (SD). Differences were analyzed using one-way analysis of variance (ANOVA) with GraphPad Prism software. $P$ values $<0.05$ was considered statistically significant.

\section{Results}

\section{Characterization of human UCB-derived EPCs and EPC-Exos}

EPCs were isolated from fresh human UCB by density gradient centrifugation. EPC colonies appeared between 7 and 21 days of culture. As viewed under the inverted microscopy, EPCs exhibited typical endothelial-like cobblestone morphology (Fig. 1A). Immunostaining (Fig. 1B) and flow cytometry analyses (Fig. 1C) showed that these cells were highly positive for CD31, CD34, CD133, vWF, VEGFR-2, and VE-cadherin, but negative for CD45. They also displayed the ability to form capillary-like structures on Matrigel, uptake ac-LDL, and bind endothelial-specific lectin UEA-1 (Fig. 1D, E). The features were consistent with the findings of previous studies $[7,20]$. All these data unequivocally confirmed that EPCs had been successfully isolated from the human UCB.

Next, we harvested EPC-Exos from the conditioned media of EPCs by differential centrifugation. TRPS analysis, TEM, and western blotting were performed to identify the purified exosomes. TRPS measurement showed that the size of EPC-Exos was approximately 50-60 nm (Fig. 2A), which was concordant with the previously reported exosomes' size distributions [12]. TEM revealed that EPC-Exos exhibited a cup- or round-shaped morphology, with a size ranging from 50 to $60 \mathrm{~nm}$ (Fig. 2B), which was consistent with the data of TRPS analysis. Western blotting indicated that these exosomes were positive for the characteristic exosomal surface marker proteins including CD9, CD63 and CD81. CD31, which is known to be present on the surface of EPCs were also detected (Fig. 2C).

\section{EPC-Exos transplantation accelerates cutaneous wound healing in STZ-induced diabetic rats}

To evaluate the effects of EPC-Exos on diabetic wound healing, full-thickness cutaneous wounds were created on the dorsal skin areas of diabetic rats which were induced by STZ injection, followed by the local injection of EPC-Exos $\left(2 \times 10^{10}\right.$ or $1 \times 10^{11}$ particles) or an equal volume of exosomes diluent (PBS) around the wound sites. As shown in Figure 3A, $\mathrm{B}$, wound closure in rat treated with EPC-Exos was significantly accelerated compared to that of PBS-treated group at day 4, 7, and 14 post-wounding. Diabetic rats receiving treatment of $1 \times 10^{11}$ particles of exosomes showed a higher wound closure rate compared with that treated with $2 \times 10^{10}$ particles of 
exosomes.

The extent of re-epithelialization, scar formation and collagen maturity are measured to evaluate the degree of wound healing and regeneration. As shown in Fig. 3C-F, the wounds treated with EPC-Exos showed markedly enhanced re-epithelialization compared to that treated with PBS at day 14 post-wounding. Moreover, narrower scars and larger collagen deposition areas were observed in wounds treatment with EPC-Exos compared with the controls. With the increase of exosomes concentration, the wound area showed higher extent of re-epithelialization and collagen maturity, and less scar formation. All these data demonstrate that exosomes treatment result in a significant acceleration of the wound healing process in diabetic rats.

\section{EPC-Exos transplantation promotes angiogenesis in the wound sites of diabetic rats}

Blood vessels are critical for repair of wound tissues. To explore whether the local transplantation of EPC-Exos can enhance new blood vessels formation during wound healing process, the wound tissues of diabetic rats receiving different treatments were excised and examined at day 14 post-wounding. As shown in Fig. 4A, blood vessels in the PBS-treated wounds were rarely observed. In contrast, a large amount of blood vessels was observed in the exosomes-treated wound sites and the number of blood vessels was enhanced with the increase of exosomes concentration.
A

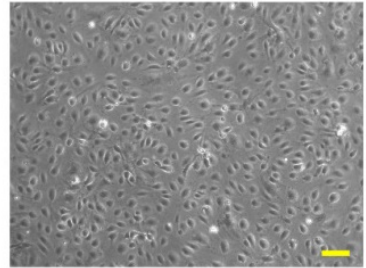

C
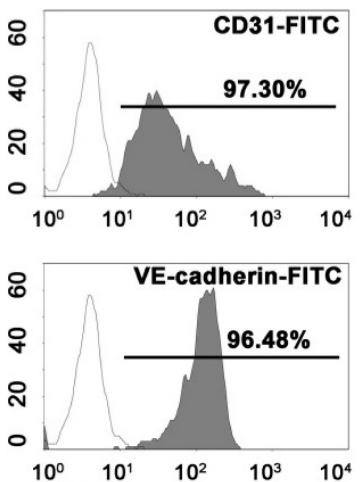

E

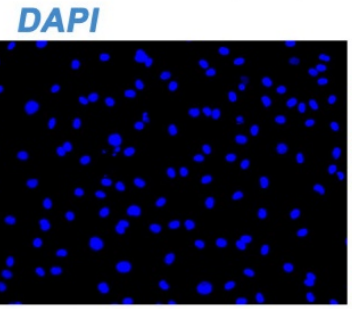

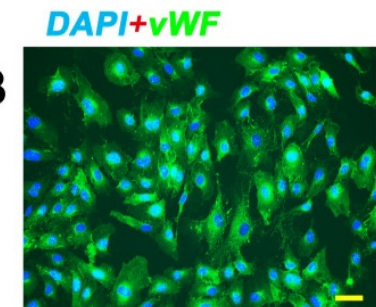

Control
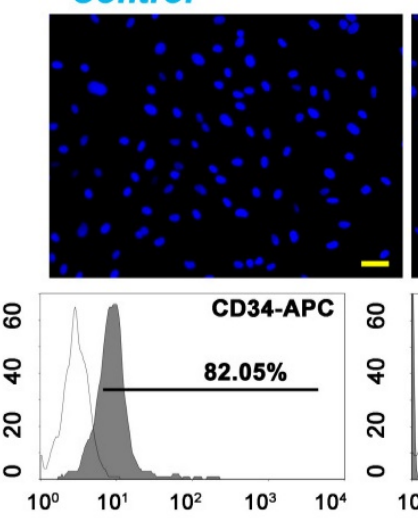

DAPI+CD34

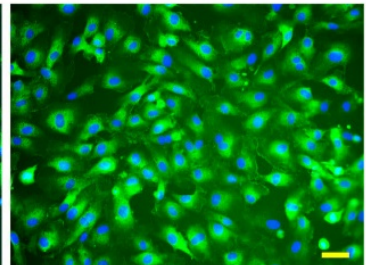

Control
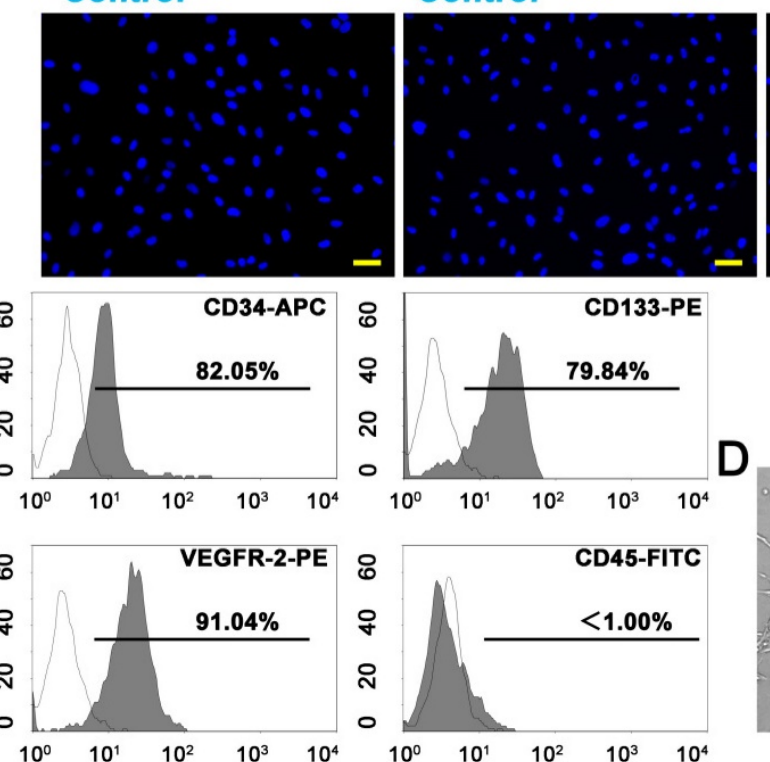

UEA-1

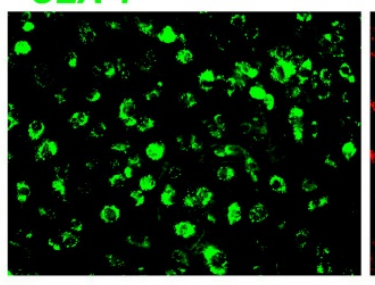

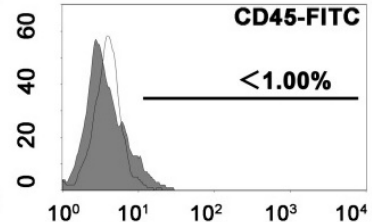

$a c-L D L$

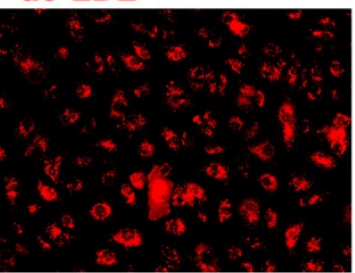

$D A P I+C D 31$

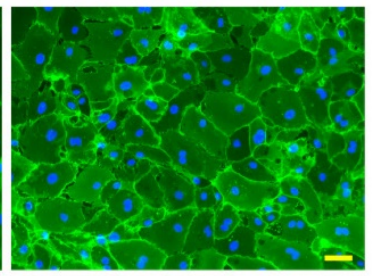

Control
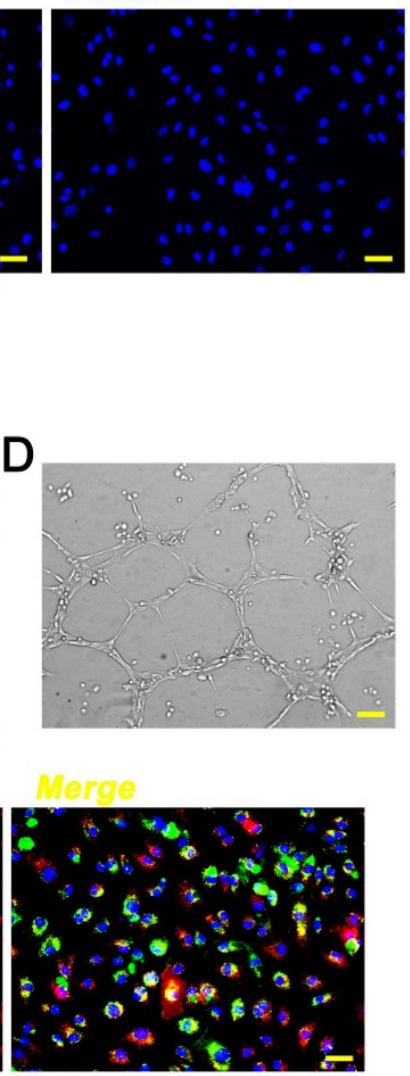

Figure 1. Isolation of endothelial progenitor cells (EPCs) from human umbilical cord blood (UCB). (A) EPCs showed a typical cobblestone-like morphology. Scale bar: $100 \mu$ m. (B) Immunostaining of cell-specific antigens on EPCs. Scale bar: $50 \mu \mathrm{m}$. (C) Flow cytometry analysis of the cell surface markers on EPCs. The isotype controls were illustrated as blank curves and the test samples were illustrated as solid gray curves. (D) EPCs formed capillary-like networks on Matrigel surfaces. Scale bar: $100 \mu \mathrm{m}$. (E) EPCs displayed ability to bind FITC-UEA-I and uptake Dil-ac-LDL. Scale bar: $50 \mu \mathrm{m}$. 


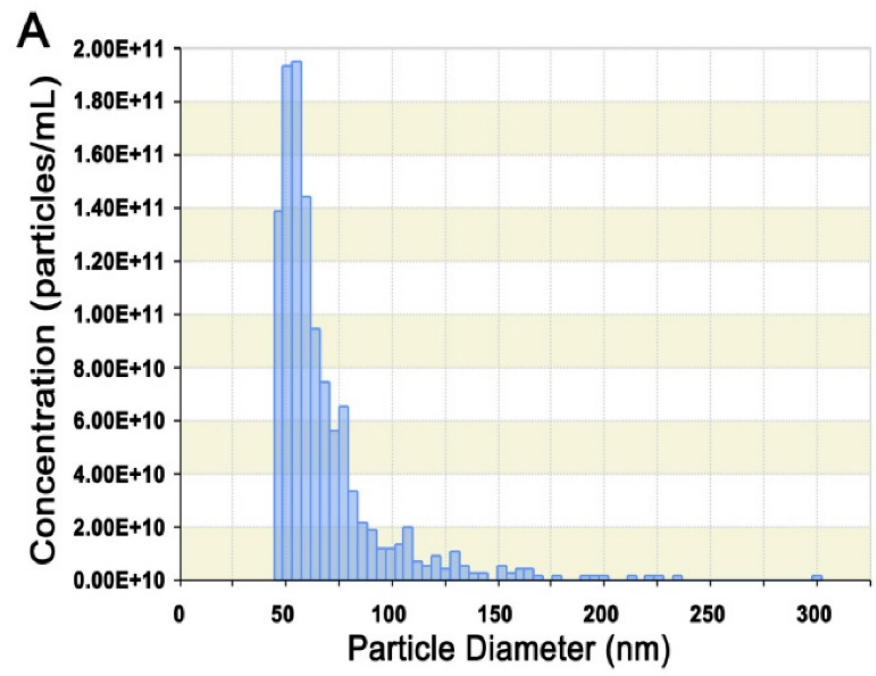

B

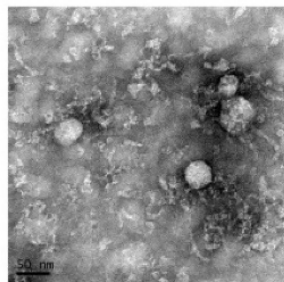

C

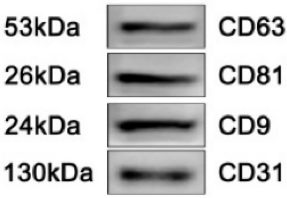

Figure 2. Characterization of exosomes released by human UCB-derived EPCs (EPC-Exos). (A) Particle size distribution and concentration of EPC-Exos measured by TRPS. (B) Morphology of EPC-Exos under a transmission electron microscopy. Scale bar: 50nm. (C) Western blotting analysis of exosomal surface marker proteins (including CD9, CD63, and CD81) and endothelial lineage cell marker (CD31) in EPC-Exos.

Blood vessels in wound sites were also evaluated by microfil perfusion. The reconstructed 3D images by micro-CT indicated that exosomes treatment induced a prominent increase in blood vessels density compared with controls and higher extent of blood vessels formation was observed in the wound sites treated with a higher dose of exosomes (Fig. 4B). The results were confirmed by a quantitative analysis of blood vessels areas at the wound sites (Fig. 4C).

The total and mature blood vessels in wound sites receiving different treatments were then identified by CD31 immunostaining and double-staining for CD31 and a-SMA, respectively (Fig. 4D, F), from which the average blood vessels density and the number of mature blood vessels were quantified (Fig. 4E, G). The results revealed that EPC-Exos treatment remarkably enhanced the amount of total and mature blood vessels in the wound sites compared to controls, and much more vessels were detected with the increase of exosomes concentration. All these data indicated that EPC-Exos could accelerate the angiogenesis and maturation of blood vessels during wound healing process. It is well established that angiogenesis makes an important contribution to postnatal new blood vessels formation. Therefore, EPC-Exos may augment the angiogenic responses of wound sites, which finally enhances the number of blood vessels in the wounds.

We performed ki67/CD31 co-staining to evaluate the effect of EPC-Exos on the proliferation of endothelial cells in the wound sites of diabetic rats. The result verified that the neovascularization induced by EPC-Exos is attributed to enhanced endothelial cells proliferation (Figure $4 \mathrm{H}$ ).

\section{Internalization of EPC-Exos by endothelial cells}

Angiogenesis refers to the formation of new blood vessels from the pre-existing vasculature through endothelial cells' proliferation, migration, and capillary tube-like structures formation [16]. Thus, we assessed the effects of EPC-Exos on the angiogenic activities of endothelial cells in vitro. Firstly, we determined whether EPC-Exos could be internalized into endothelial cells. EPCs were labeled by a green fluorescent lipophilic dye $(\mathrm{DiO})$ and then the secreted exosomes were harvested. After incubating HMECs with the labeled exosomes for 2 hours, the DiO-labeled exosomes were transferred to the perinuclear region of HMECs (Fig. 5A), indicating that the EPC-Exos were incorporated into HMECs.

\section{Pro-angiogenic effects of EPC-Exos on endothelial cells}

To explore the functional role of EPC-Exos in angiogenesis, HMECs were cultured in serum-free MCDB131 media containing exosomes $\left(2 \times 10^{10}\right.$ or $1 \times$ $10^{11}$ particles $/ \mathrm{mL}$ ) or an equal volume of PBS for a series of in vitro angiogenesis-related assays. The effect of EPC-Exos on the proliferation of HMECs was examined by CCK- 8 cell counting analysis. The results indicated that $2 \times 10^{10}$ particles/mL of EPC-Exos were able to significantly enhance the proliferation of HMECs, and HMECs treated with a higher dose of exosomes $\left(1 \times 10^{11}\right.$ particles $\left./ \mathrm{mL}\right)$ showed much higher proliferative capability (Fig. 5B). 
A Control Exos $2 \times 10^{10}$ particles Exos $1 \times 10^{11}$ particles
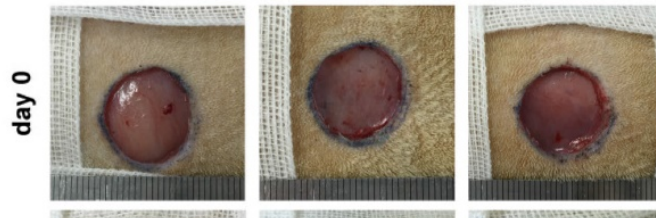

B
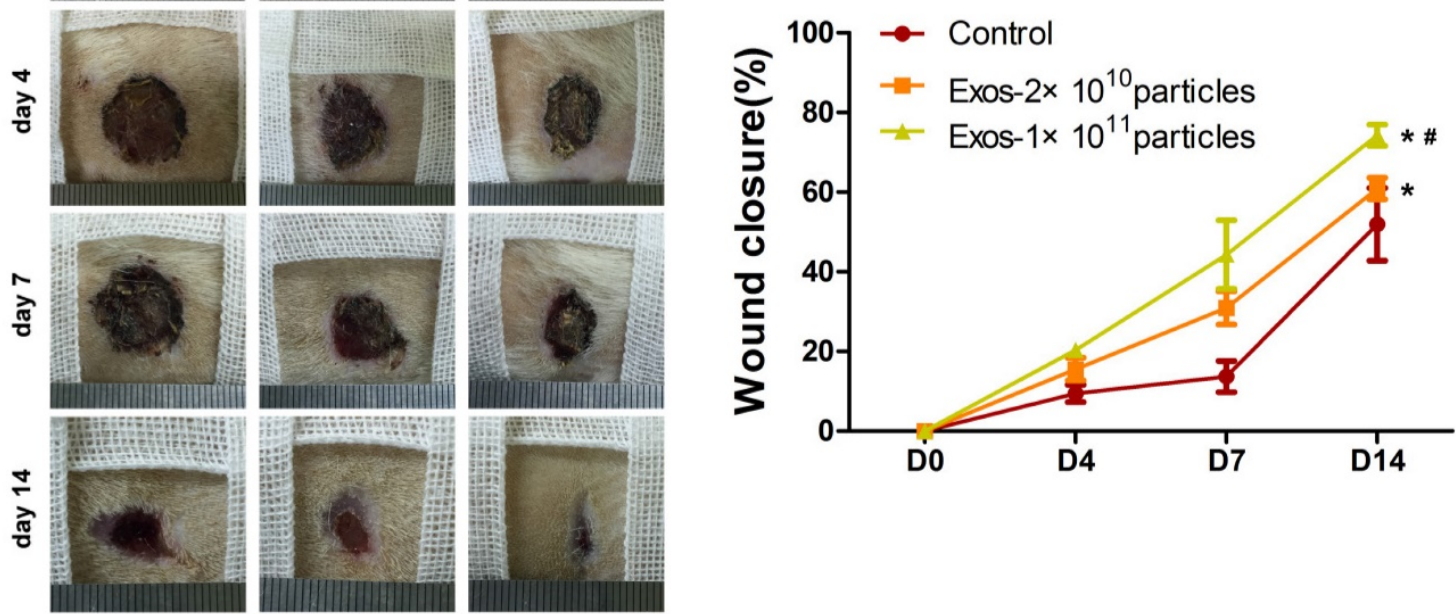

C

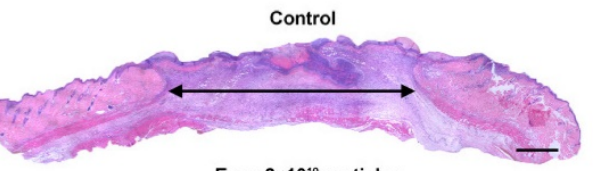

Exos $2 \times 10^{10}$ particles

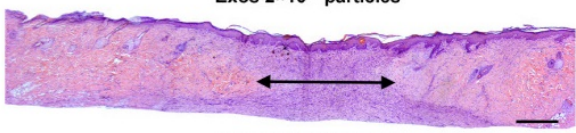

Exos $1 \times 10^{11}$ particles

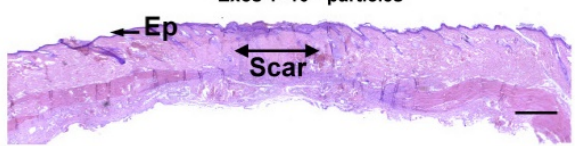

D

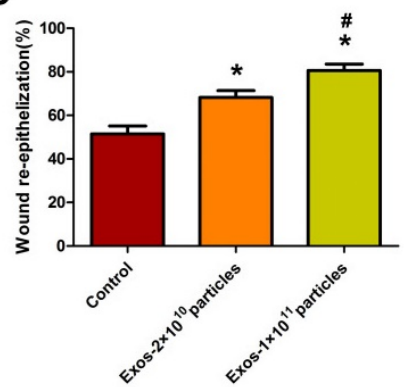

Exos $2 \times 10^{10}$ particles

$\mathbf{F}$
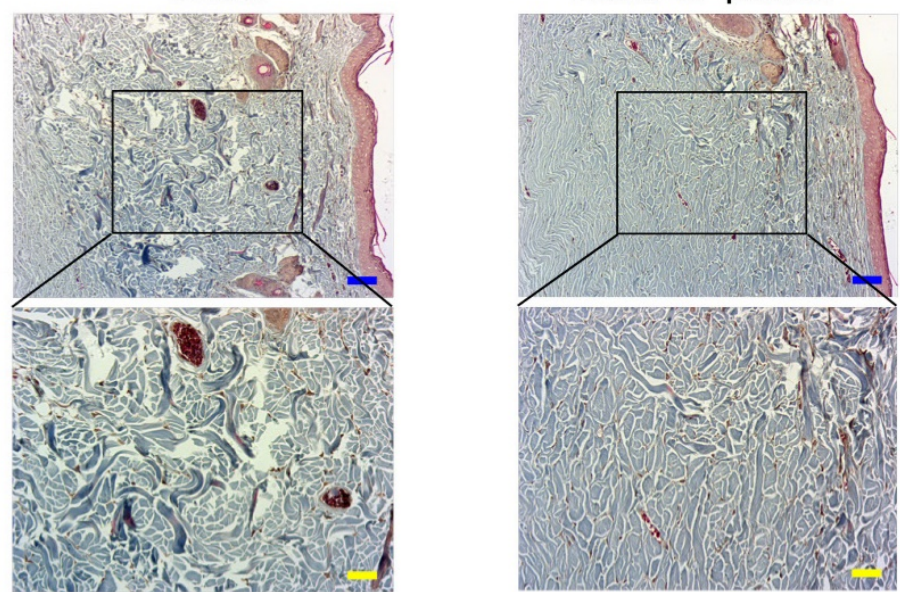

E

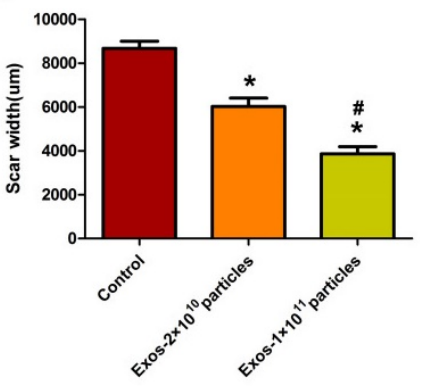

Exos $1 \times 10^{11}$ particles

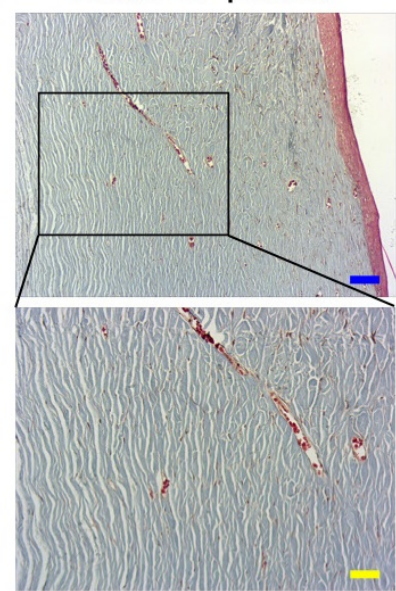

Figure 3. EPC-Exos transplantation accelerates cutaneous wound healing in diabetic rats. (A) Gross view of wounds treated with PBS or different concentration of EPC-Exos at day 4, 7, and 14 post-wounding. (B) The rate of wound-closure on different days in wounds receiving different treatments. (C) $\mathrm{H} \& \mathrm{E}$ staining of wound sections after treatment with PBS or different concentration of EPC-Exos at 14 days post-wounding. The double-headed arrows indicate the edges of the scar. Scale bar: 2 mm. The extent of re-epithelialization (D) and widths of the scars (E) was evaluated in PBS or EPC-Exos-treated wounds at 14 days post-wounding. Ep, Epithelium. (F) Evaluation of collagen maturity by Masson's trichrome staining. Blue Scale bar: $100 \mu \mathrm{m}$. Yellow scale bar: $50 \mu \mathrm{m}$. $(* P<0.05$ compared with the PBS group (control), \#P<0.05 compared with the EPC-Exos $2 \times 10^{10}$ particles group.) 


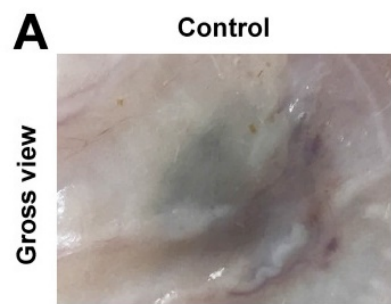

B

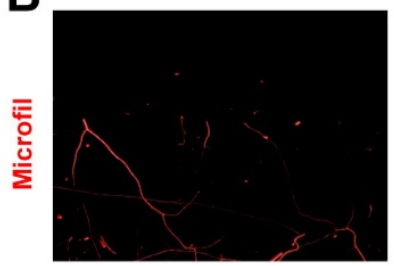

D

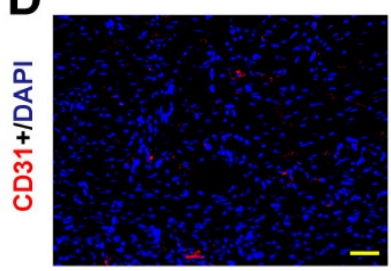

F

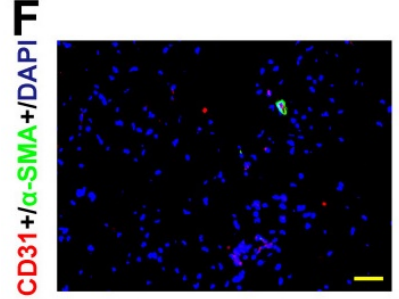

H

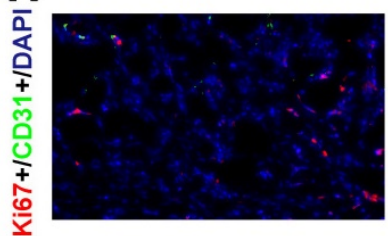

Exos $2 \times 10^{10}$ particles
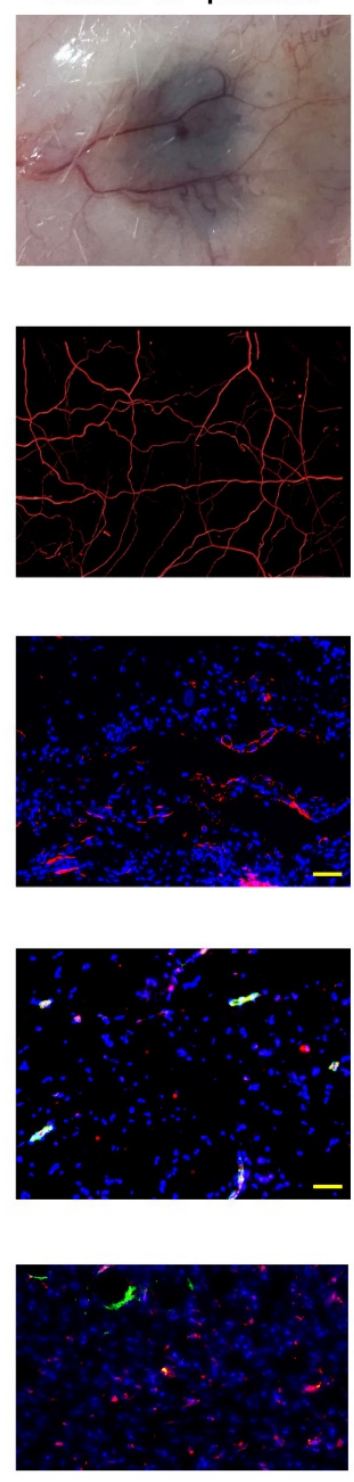

\section{Exos $1 \times 10^{11}$ particles}
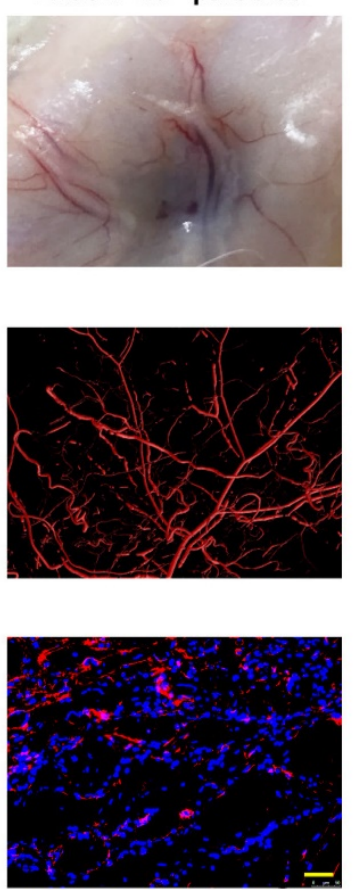

C

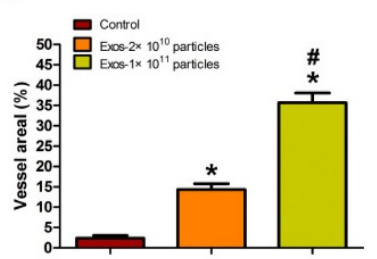

E

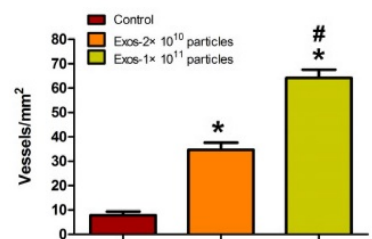

G
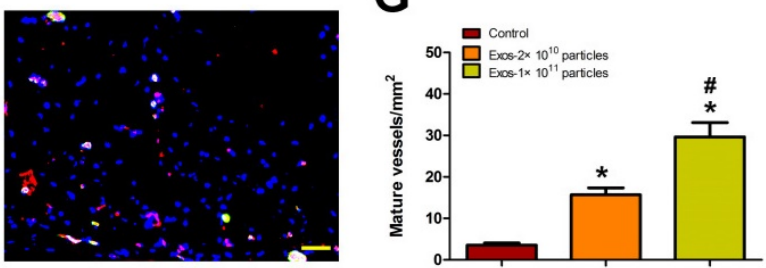

Figure 4. EPC-Exos transplantation promotes new blood vessels formation in the wound sites of diabetic rats. (A) Gross view of blood vessels in wounds treated with PBS or different concentration of EPC-Exos at day 14 post-wounding. Blood vessels density was determined by microfil perfusion. The reconstructed 3D images by micro-CT were illustrated in (B). (C) Quantitative analysis of the vascularized area in wounds receiving different treatments. (D) Immunofluorescence staining for CD31 in wounds after

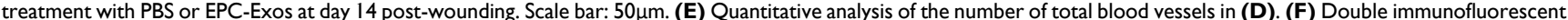
staining for CD31 and SMA in wounds receiving different treatments at day 14 post-wounding. Scale bar: $50 \mu \mathrm{m}$. (G) Quantitative analysis of the number of mature blood vessels in (F). (H) Double immunofluorescent staining for CD31 and Ki67 in wounds to identify the proliferating cells. $(* P<0.05$ compared with the PBS group (control), $\# P<0.05$ compared with the EPC-Exos $2 \times 10^{10}$ particles group.)

To assess the effect of EPC-Exos on the angiogenic tubule formation of endothelial cells, HMECs were seeded on Matrigel and cultured with exosomes or PBS for 4 hours and 8 hours. The total branching points, total tube length, cell covered area, and total loops at the indicated time were measured to quantify the ability of HMECs to form tubes. As shown in Fig. 5C, D, all the indicators were increased in exosomes-treated HMECs, and better tube formation was observed with the increase of exosomes concentration. A scratch wound assay was used to determine the effect of EPC-Exos on the migration of HMECs. The result showed that exosomes treatment remarkably enhanced the motility of HMECs and the migration ability of HMECs was further enhanced after treatment with a higher dose of exosomes (Fig. 5E, F). Taken together, our in vitro functional assays revealed that EPC-Exos could activate a series of angiogenic responses of recipient endothelial cells. Because the high dose of exosomes $\left(1 \times 10^{11}\right.$ particles $\left./ \mathrm{mL}\right)$ could induce more significant pro-angiogenic and/or wound healing effects on target tissues and cells than the lower dose of exosomes $\left(2 \times 10^{10}\right.$ particles $\left./ \mathrm{mL}\right)$, the concentration of $1 \times 10^{11}$ particles $/ \mathrm{mL}$ was used for the following experiments. 
A

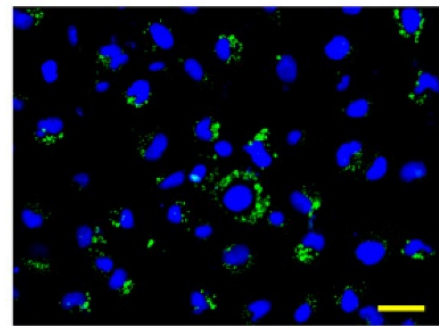

C

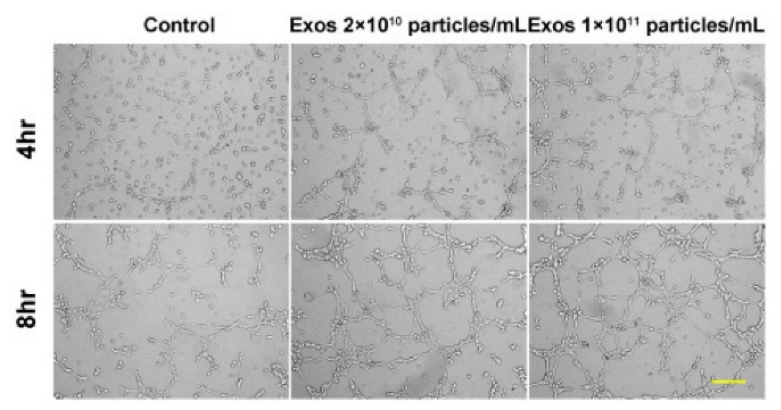

E

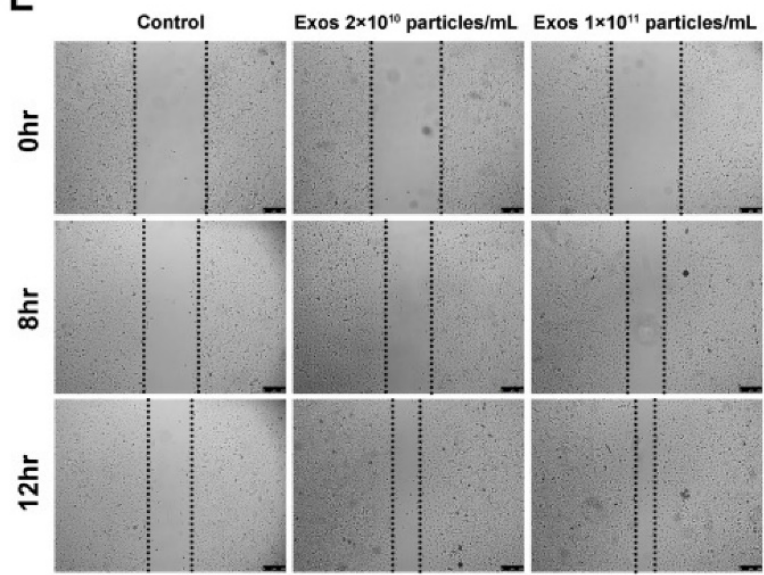

B

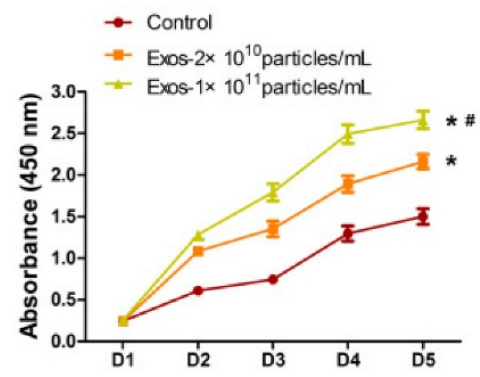

D

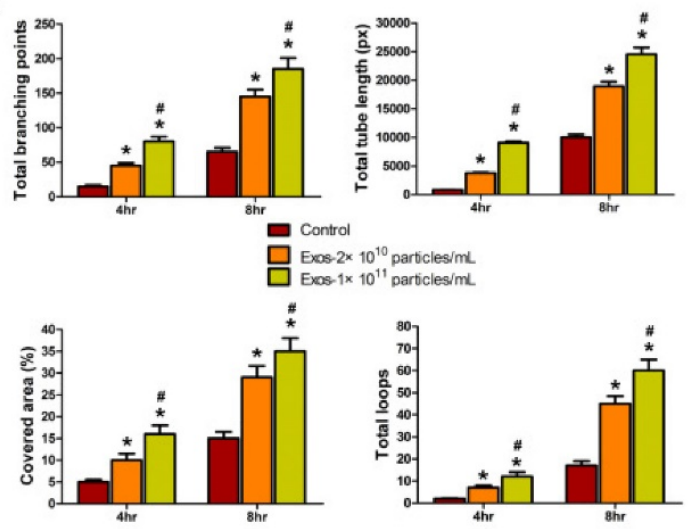

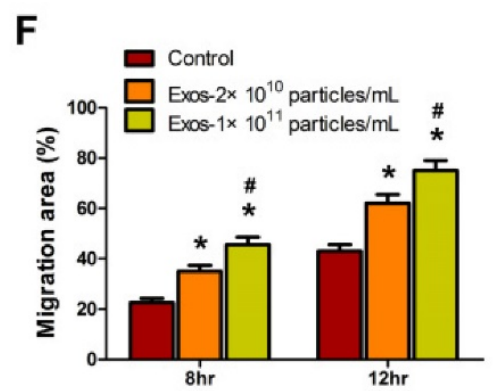

Figure 5. Internalization of EPC-Exos into endothelial cells and their pro-angiogenic effects on recipient endothelial cells. (A) Fluorescent microscopy analysis of DiO-labeled EPC-Exos internalization by human microvascular endothelial cells (HMECs). The green-labeled exosomes were visible in the perinuclear region of HMECs. Scale bar: $50 \mu m$. (B) EPC-Exos promoted HMECs proliferation as analyzed by Cell Counting Kit-8 assay, and HMECs treated with a higher dose of exosomes showed much higher proliferative capability. (C) The tube formation capability was increased in HMECs stimulated with EPC-Exos, and better tube formation was observed with the increase of exosomes concentration. Scale bar: $100 \mu \mathrm{m}$. (D) The total branching points, total tube length, cell covered area, and total loops at the indicated time were measured to quantify the ability of HMECs to form tubes. (E) EPC-Exos augmented the motility of HMECs, and the pro-migratory effect of EPC-Exos on HMECs was enhanced with a higher concentration of exosomes. Scale bar: $250 \mu \mathrm{m}$. (F) Quantitative analysis of the migration rates in (E). ( ${ }^{*} P<0.05$ compared with the PBS group (control), $\# P<0.05$ compared with the EPC-Exos $2 \times 10^{10} \mathrm{particles} / \mathrm{mL}$ group.)

\section{The activation of Erk1/2 signaling in endothelial cells in response to EPC-Exos stimulation}

To investigate the mechanism through which EPC-Exos modulate endothelial cells function, microarray was performed to analyze the gene expression of HMECs treated with exosomes or an equal volume of PBS. Differentially expressed genes (DEGs) were identified with a cutoff of absolute fold change $\geq 1.5$ and $p$ value $<0.05$. The results showed that 1958 genes were differently expressed between the exosomes- treated HMECs and control groups
(Fig. 6A). Among them, 786 genes were up-regulated and 1172 genes were down-regulated following exosomes stimulation. Then, the DEGs were biologically interpreted and their involved regulatory signaling pathways were further explored. The results showed that exosomes treatment markedly altered the levels of a class of genes involved in Erk1/2 signaling pathway. Among them, the upstream molecules which positively regulate Erk1/2 signaling such as fibroblast growth factor 2 (FGF-2), interleukin 6 (IL-6), and IL-8, were profoundly up-regulated after exosomes stimulation. The downstream target genes of this pathway such as Id1, Cox-2, VEGFA, c-Myc, 
and cyclin D1 (CCND1), were also significantly increased after exosomes treatment. Our results suggest that incubation with EPC-Exos might result in the activation of Erk1/2 signaling in HMECs.

Next, qRT-PCR analyses were performed to confirm the expression of these Erk1/2 signaling-related DEGs. The results demonstrated that the levels of FGF-2, IL-6, IL-8, c-Myc, Id1, Cox-2, and VEGFA were markedly enhanced in HMECs after exosomes stimulation (Fig. 6B), which was consistent with the data of microarray analyses. No significant difference was observed in the expression of CCND1 between the exosomes-treated and control groups (Fig. 6B).

To determine whether Erk1/2 signaling is activated after exosomes treatment, HMECs were treated with EPC-Exos or PBS for 24 hours and the proteins were harvested to assess the phosphorylation of Erk1/2 using western blotting (Fig. 6C). The results revealed that EPC-Exos stimulation induced a significant increase of Erk1/2 phosphorylation on both Thr202 and Tyr204 residues, indicating that Erk1/2 signaling in HMECs was activated by

A

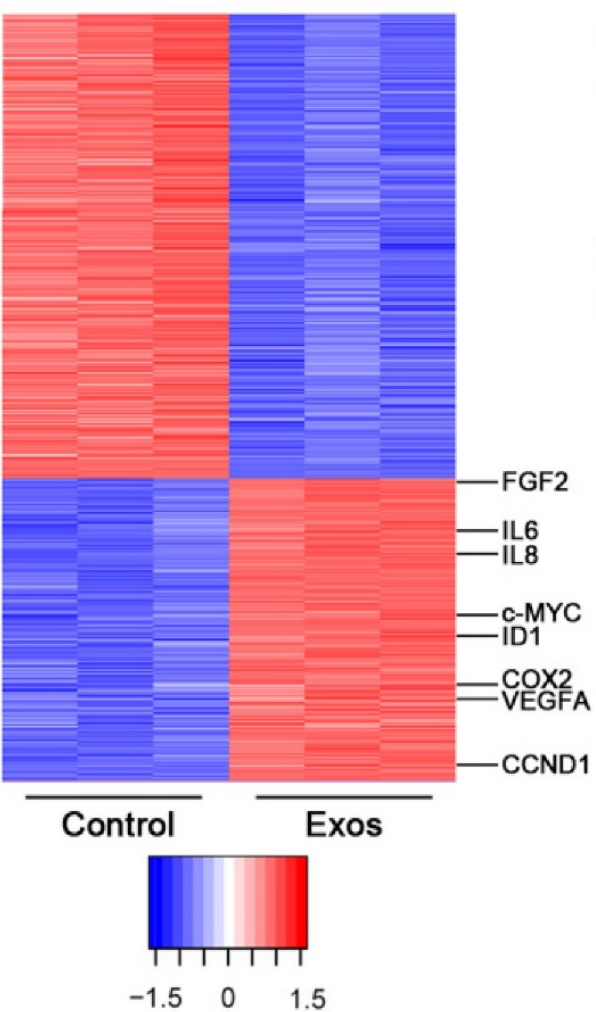

exosomes. Moreover, the levels of molecules involved in this signaling process including c-Myc, Id1, Cox-2, and VEGFA, were also remarkably up-regulated in exosomes-treated HMECs compared with controls, which further confirmed that the Erk1/2 signaling was activated after exosomes stimulation.

\section{Erk1/2 signaling mediated the EPC-Exos-induced pro-angiogenic effects on endothelial cells}

To determine whether the activation of Erk1/2 signaling is the underlying mechanism by which EPC-Exos regulate endothelial cells function, HMECs were pre-treated with the MEK1/2 inhibitor U0126 for 2 hours and then cultured with EPC-Exos or PBS for 24 hours. qRT-PCR and western blotting were performed to detect the mRNA and protein levels of Erk1/2 signaling-related molecules. As shown in Fig. $7 \mathrm{~A}, \mathrm{~B}$, the increase of phosphorylation of Erk1/2 and the expression of the related molecules induced by exosomes was significantly inhibited by U0126 pre-incubation, suggesting that the exosomes-mediated Erk1/2 signaling activation could be abolished by U0126. A series of

B
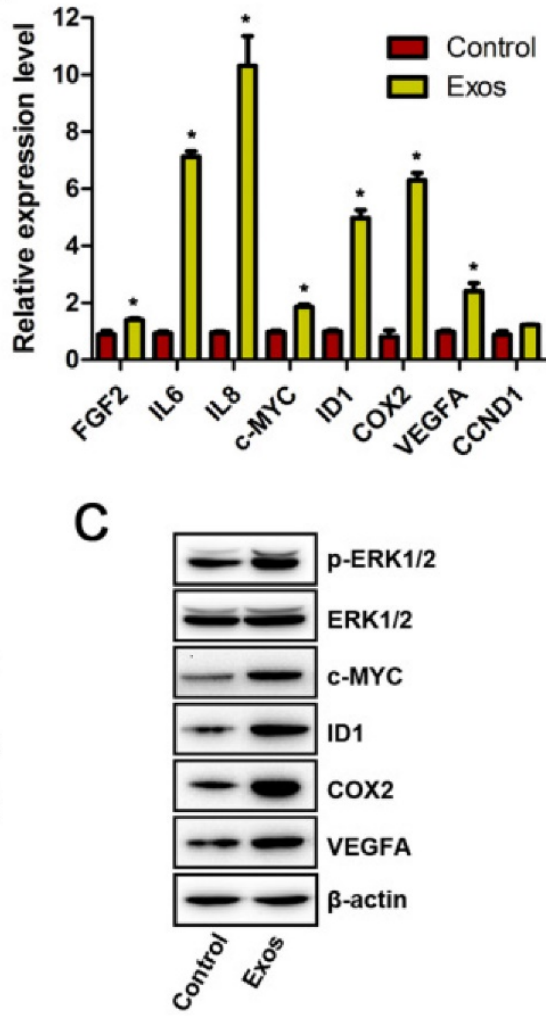

Figure 6. Erk1/2 signaling was activated in HMECs after EPC-Exos treatment. (A) The differentially expressed genes (DEGs) in HMECs in response to EPC-Exos stimulation were illustrated as a heat map. A p-value cut-off of 0.05 and a fold value change of $\geq 1.5$ were used as a filter to identify the DEGs. (B) The altered expression of Erk1/2 signaling-related genes was confirmed by QRT-PCR analyses. (C) EPC-Exos enhanced the phosphorylation of Erk1/2 and the protein levels of a class of angiogenesis-related molecules downstream of Erk1/2 pathway. ( $* P<0.05$ compared with the control group) 
angiogenesis-related assays were performed to confirm the role of Erk1/2 signaling in EPC-Exos-mediated pro-angiogenic effects on HMECs. CCK-8 assay showed that the exosomes-induced increase in the proliferation HMECs was markedly blocked by U0126 (Fig. 7C). The tube formation assay on Matrigel is an in vitro model of angiogenesis. As shown in Fig. 7D, U0126 incubation could profoundly inhibit the positive effect of EPC-Exos on the tube structures formation of HMECs. The exosomes-induced increase of the indicators including total branching points, total tube length, cell covered area, and total loops that evaluate the ability of HMECs to form tubes were significantly reduced by U0126 (Fig. 7E). The results of scratch wound assay demonstrated that the pro-migratory effect induced by EPC-Exos could be dramatically abolished by U0126 (Fig. 7F, G).
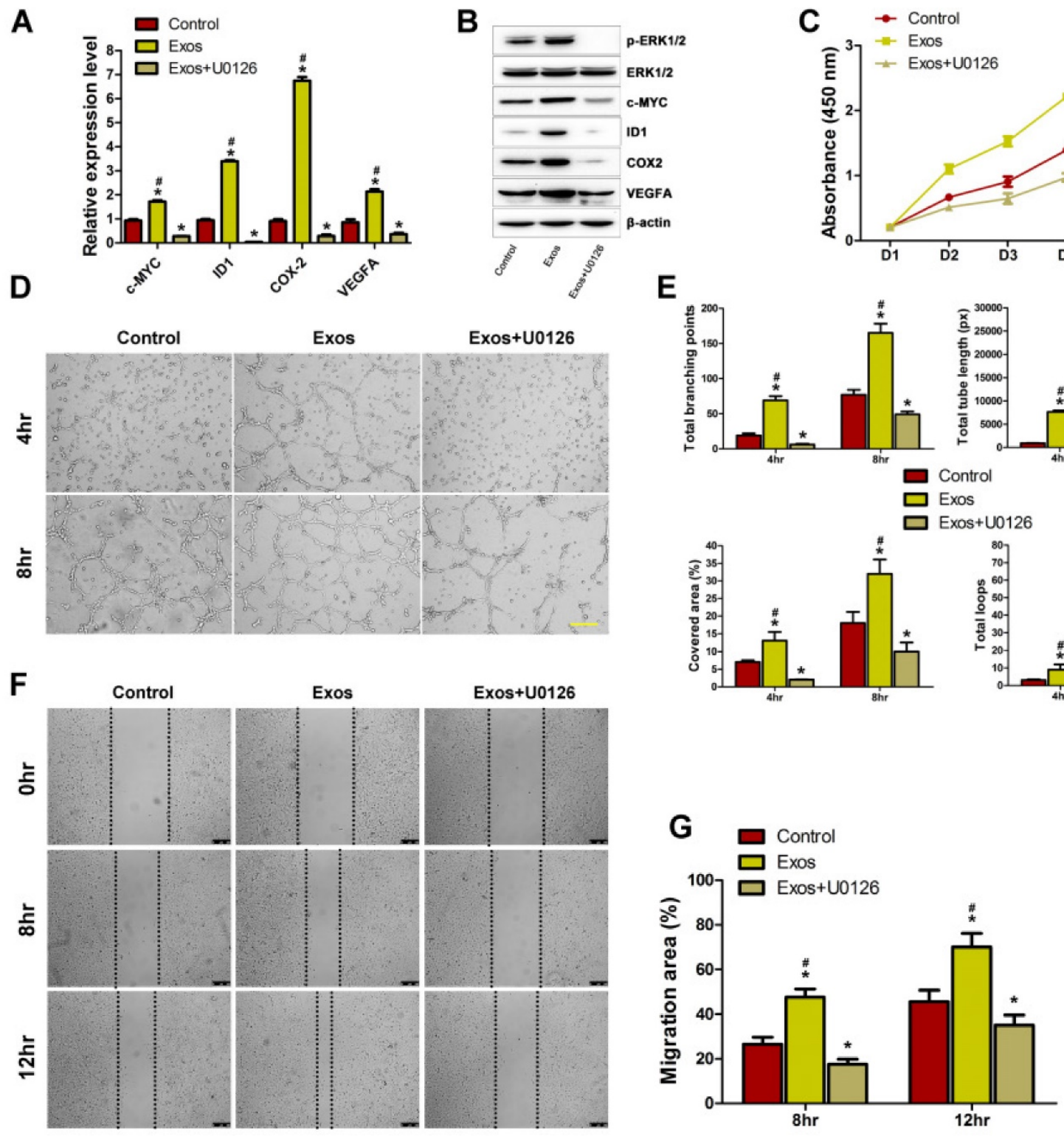

Figure 7. Erk1/2 signaling mediated the EPC-Exos-induced pro-angiogenic effects on HMECs. EPC-Exos increased the mRNA (A) and protein levels (B) of Erk1/2 signaling-related molecules, and their up-regulation induced by EPC-Exos was abolished by a specific Erk1/2 signaling inhibitor (U0126; $10 \mu M)$. (C) The proliferation of HMECs was remarkably enhanced after EPC-Exos treatment, whereas this effect was blocked by U0126. (D) EPC-Exos increased the tube formation ability of HMECs, but this effect was inhibited by $\mathrm{U} 0126$. Scale bar: $100 \mu \mathrm{m}$. (E) The total branching points, total tube length, cell covered area, and total loops at the indicated time were assessed to quantify the ability of HMECs to form tubes at each time point. (F) EPC-Exos induced a remarkable increase in the motility of HMECs, but the pro-migratory effect was dramatically decreased by U0126. Scale bar: $250 \mu \mathrm{m}$. (G) Quantitative analysis of the migration rates in (F). ( $* P<0.05$ compared with the control group, $\# P<0.05$ compared with the EPC-Exos $+U 0126$ group.) 
Collectively, our data indicate that the pro-angiogenic effects of EPC-Exos on HMECs are mainly mediated by the activation of Erk $1 / 2$ signaling pathway. Studies have demonstrated that c-Myc is a downstream target of Erk1/2 signaling and can activate cell proliferation by controlling the transcription of key cell cycle regulators, including by suppressing the expression of cyclin-dependent kinase inhibitor 1A (CDKN1A) (p21 WAF1/CIP1) and CDKN1B (p27Kip1). In our study, we found that EPC-Exos could induce the expression of the cell cycle activator c-Myc and enhance the proliferation of endothelial cells by activating Erk1/2 signaling. Further microarray and qRT-PCR analyses showed that EPC-Exos treatment resulted in significant decreases in the levels of CDKN1A and CDKN1B in endothelial cells, whereas this effect was markedly blocked by the Erk1/2 signaling specific inhibitor U0126 (Fig. S1). Subsequently, after incubation with EPC-Exos for 24 hours, we evaluated the changes in cell cycle of endothelial cells by flow cytometry with the intercalating dye propidium iodide (PI). The result showed that EPC-Exos stimulation induced a remarkable increase of cells at $S$ stage compared to control group, and an additional treatment with U0126 markedly abolished the exosomes-induced positive effect on G1 to S-phase transition of endothelial cells (Fig. S1). Taken together, our data suggest that the promotion of S-phase entry may be the downstream mechanism by which EPC-Exos enhance the proliferation of endothelial cells via Erk1/2 signaling.

\section{Discussion}

Acute cutaneous wounds caused by trauma can become chronic non-healing wounds in patients with diabetes mellitus [21]. Although numerous therapeutic attempts have been made to accelerate the healing process of diabetic wounds, the optimal treatment strategies are still being developed. In this study, we provided the first demonstration that the topical treatment of EPC-Exos, the biomolecules released by EPCs, could promote angiogenesis and cutaneous wound healing in STZ-induced diabetic rats. We also found that EPC-Exos markedly enhanced the angiogenic activity of endothelial cells in vitro and further demonstrated that Erk1/2 signaling was the critical mediator during this process. Our results suggest that EPC-Exos enhance angiogenic responses of endothelial cells by activating Erk1/2 signaling, which finally facilitates cutaneous wound repair and regeneration.

In the present study, UCB was used for isolation and culture of EPCs. Then the EPC-Exos was applied to repair diabetic chronic wounds. UCB can be collected during labor, with the distinct advantages of no risk to the donors, minimal ethical concerns, a low incidence of graft-versus-host disease and easy accessibility [22]. UCB-derived MSCs have already been transplanted to improve islet function in patients with T2D [23]. In particular, it is reported that MSC-derived exosomes have already been used to treat therapy-refractory graft-versus-host disease in clinic [24]. In addition, UCB also contains abundant progenitor cells, so it is good source for clinic therapy. Hence, the clinical translation of hUCB-EPCs-derived exosomes on the therapeutic effect of refractory cutaneous ulcer has a very good prospect

To our knowledge, this is the first study to demonstrate that the local transplantation of EPC-Exos into the full-thickness excisional skin wounds of diabetic rats could induce significant regenerative effects in the wound sites, as defined by a more rapid wound closure, higher re-epithelialization and collagen deposition, and less scar formation. Moreover, we showed that exosomes treatment remarkably enhanced the amount of total and mature blood vessels in the wound sites. With the increase of exosomes concentration, the exosomes-mediated beneficial effects on blood vessels formation and cutaneous wound repair were further enhanced. The formation of new blood vessels in postnatal life occurs mainly via angiogenesis [25], which is essential for the survival, repair, and remodeling of wounded tissues. Thus, EPC-Exos may augment angiogenesis in the wound sites, which enhances the blood vessels density and thereby accelerate the diabetic wound healing process. Since angiogenesis involves the migration, proliferation, and angiogenic tubule formation of endothelial cells [16], we then evaluated the effects of EPC-Exos on the behavior of endothelial cells in vitro. The results revealed that EPC-Exos could be incorporated into endothelial cells and significantly enhance the angiogenic activities of endothelial cells, which further confirmed the pro-angiogenic property of EPC-Exos.

Exosomes are an integral part of the intercellular microenvironment and represent an important mode of cell-to-cell communication. They can serve as a vehicle to transfer membrane receptors, proteins, mRNAs, and microRNAs to recipient cells and thereby alter the gene expression and protein translation of the recipient cells to modulate the bioactivity of target cells [26]. For example, Shabbir et al [27] demonstrated that the exosomes released by human bone marrow-derived MSCs could induce the activation of several signaling pathways in target fibroblasts, including Akt, Erk1/2, and STAT3. Zhang et al [28] reported that the exosomes released by 
human UCB-derived MSCs shuttled Wnt4 protein and could induce $\beta$-catenin nuclear translocation and enhance the activity of recipient skin cells. In this study, by using microarray and qRT-PCR analyses, we found that the endothelial cells stimulated by EPC-Exos showed higher mRNA levels of a network of genes that are known to be involved in Erk1/2 pathway, a signaling cascade that plays crucial roles in wound healing, including roles in cell proliferation, migration, and angiogenesis [29-31]. Among these significantly altered genes, FGF-2, IL-6, and IL-8 are upstream modulators of Erk1/2 signaling and can induce a variety of cellular angiogenic responses by activating this signaling process [32-34]. Id1, Cox-2, VEGFA, and c-Myc are a class of angiogenesis-related molecules that can be regulated by Erk1/2 signaling at the mRNA level [35-38]. The results indicated that Erk1/2 signaling in endothelial cells might be activated after exosomes stimulation. The hypothesis was confirmed by a significant up-regulation in the levels of $\mathrm{p}$-Erk1/2 and the molecules downstream to this signaling in endothelial cell treated by exosomes. Moreover, we found that the activation of these Erk1/2 signaling-related molecules and the pro-angiogenic effects induced by EPC-Exos could be profoundly reduced by the specific Erk1/2 signaling inhibitor U0126, which suggests that Erk1/2 signaling is essential for the exosomes-induced angiogenic responses of endothelial cells. Several studies have proposed that the transfer of exosomal miRNAs may modulate the biological functions of the acceptor cells [39-45]. For example, exosomal miR-223 through down-regulation of Sema3A and Stat3 signal pathway contributes to MSCs-mediated cardio-protection in sepsis [46]. Mineralizing osteoblasts-derived exosomes contain miRNAs may through activating Wnt signaling pathway promote MSCs osteogenic differentiation [47]. Recent studies showed that miR-21 could induce angiogenesis through activating Erk1/2 signaling pathway [48]. In particular, studies suggested that miR-21 is enriched in human EPC-derived exosomes [49], and we also confirmed the result by qPCR analysis (data not show). So we speculate that the transfer of miR-21-loaded exosomes from hUCB-EPCs to endothelial cells may contribute to the activation of Erk1/2 signaling pathway, and subsequently promote angiogenesis. The exosomes' role is likely to be horizontal transfer of cellular information; exosomes can be considered as mediators of intercellular communication. The exact mechanism still needs further investigation.

\section{Conclusion}

In summary, we firstly demonstrated that EPC-Exos could effectively promote new blood vessels formation and cutaneous wound healing in diabetic rats. The underlying mechanism may be the activation of angiogenic responses of endogenous endothelial cells in the wound sites, as EPC-Exos could enhance the proliferation, migration, and tube formation of endothelial cells in vitro. Moreover, in the pro-angiogenic effects of EPC-Exos on endothelial cells, Erk1/2 signaling plays a critical role since inhibition of this signaling process significantly blocked the pro-angiogenic effects of exosomes. These results suggest that the use of EPC-Exos may represent a promising strategy for diabetic cutaneous wound healing.

\section{Supplementary Material}

Supplementary figure S1.

http://www.ijbs.com/v12p1472s1.pdf

\section{Acknowledgments}

This study was financially supported by the National Natural Science Foundation of China (Grant No.: 81472152, 81572223 and 81572120) and Joint Project Funding for Major Diseases in Shanghai (Grant NO.: 2014ZYJ B0301).

\section{Competing Interests}

The authors have declared that no competing interest exists.

\section{References}

1. Kharroubi AT, Darwish HM: Diabetes mellitus: The epidemic of the century. World J Diabetes 2015, 6:850-867.

2. Lioupis C: Effects of diabetes mellitus on wound healing: an update. JWound Care 2005, 14:84-86.

3. Kant V, Gopal A, Kumar D, Pathak NN, Ram M, Jangir BL, Tandan SK, Kumar D: Curcumin-induced angiogenesis hastens wound healing in diabetic rats. J Surg Res 2015, 193:978-988.

4. Kota SK, Meher LK, Jammula S, Kota SK, Krishna SV, Modi KD: Aberrant angiogenesis: The gateway to diabetic complications. Indian J Endocrinol Metab 2012, 16:918-930.

5. Kim JY, Song SH, Kim KL, Ko JJ, Im JE, Yie SW, Ahn YK, Kim DK, Suh W: Human cord blood-derived endothelial progenitor cells and their conditioned media exhibit therapeutic equivalence for diabetic wound healing. Cell Transplant 2010, 19:1635-1644

6. Ackermann M, Pabst AM, Houdek JP, Ziebart T, Konerding MA: Priming with proangiogenic growth factors and endothelial progenitor cells improves revascularization in linear diabetic wounds. Int J Mol Med 2014, 33:833-839.

7. Critser PJ, Yoder MC: Endothelial colony-forming cell role in neoangiogenesis and tissue repair. Curr Opin Organ Transplant 2010, 15:68-72.

8. Duscher D, Barrera J, Wong VW, Maan ZN, Whittam AJ, Januszyk M, Gurtner GC: Stem Cells in Wound Healing: The Future of Regenerative Medicine? A Mini-Review. Gerontology 2015.

9. Herberts CA, Kwa MS, Hermsen HP: Risk factors in the development of stem cell therapy. J Transl Med 2011, 9:29.

10. Kansu E: Thrombosis in stem cell transplantation. Hematology 2012, 17 Suppl 1:S159-162.

11. Zhang M, Malik AB, Rehman J: Endothelial progenitor cells and vascular repair. Curr Opin Hematol 2014, 21:224-228

12. De Jong OG, Van Balkom BW, Schiffelers RM, Bouten CV, Verhaar MC: Extracellular vesicles: potential roles in regenerative medicine. Front Immunol 2014, 5:608.

13. Burger D, Vinas JL, Akbari S, Dehak H, Knoll W, Gutsol A, Touyz RM, Allan DS, Burns KD: Human Endothelial Colony-Forming Cells Protect against Acute Kidney Injury: Role of Exosomes. Am J Pathol 2015.

14. Xin H, Li Y, Chopp M: Exosomes/miRNAs as mediating cell-based therapy of stroke. Front Cell Neurosci 2014, 8:377. 
15. Huang L, Ma W, Ma Y, Feng D, Chen H, Cai B: Exosomes in mesenchymal stem cells, a new therapeutic strategy for cardiovascular diseases? Int J Biol Sci 2015, 11:238-245.

16. Yoo SY, Kwon SM: Angiogenesis and its therapeutic opportunities. Mediators Inflamm 2013, 2013:127170.

17. Kuang L, Feng J, He G, Jing T: Knockdown of Nrf2 inhibits the angiogenesis of rat cardiac micro-vascular endothelial cells under hypoxic conditions. Int J Biol Sci 2013, 9:656-665.

18. Ram M, Singh V, Kumawat S, Kumar D, Lingaraju MC, Uttam Singh T, Rahal A, Kumar Tandan S, Kumar D: Deferoxamine modulates cytokines and growth factors to accelerate cutaneous wound healing in diabetic rats. Eur I Pharmacol 2015, 764:9-21.

19. Ades EW, Candal FJ, Swerlick RA, George VG, Summers S, Bosse DC, Lawley TJ: HMEC-1: establishment of an immortalized human microvascular endothelial cell line. J Invest Dermatol 1992, 99:683-690.

20. Basile DP, Yoder MC: Circulating and tissue resident endothelial progenitor cells. J Cell Physiol 2014, 229:10-16.

21. Idrovo JP, Yang WL, Jacob A, Ajakaiye MA, Cheyuo C, Wang Z, Prince JM, Nicastro J, Coppa GF, Wang P: Combination of adrenomedullin with its binding protein accelerates cutaneous wound healing. PLoS One 2015, 10:e0120225

22. He B, Li X, Yu H, Zhou Z: Therapeutic potential of umbilical cord blood cells for type 1 diabetes mellitus. J Diabetes 2015, 7:762-773.

23. Guan LX, Guan H, Li HB, Ren CA, Liu L, Chu JJ, Dai LJ: Therapeutic efficacy of umbilical cord-derived mesenchymal stem cells in patients with type 2 diabetes. Exp Ther Med 2015, 9:1623-1630.

24. Kordelas L, Rebmann V, Ludwig AK, Radtke S, Ruesing J, Doeppner TR, Epple M, Horn PA, Beelen DW, Giebel B: MSC-derived exosomes: a novel tool to treat therapy-refractory graft-versus-host disease. Leukemia 2014, 28:970-973.

25. Bulgin D: Therapeutic Angiogenesis in Ischemic Tissues by Growth Factors and Bone Marrow Mononuclear Cells Administration: Biological Foundation and Clinical Prospects. Curr Stem Cell Res Ther 2015, 10:509-522.

26. Camussi G, Deregibus MC, Bruno S, Cantaluppi V, Biancone L: Exosomes/microvesicles as a mechanism of cell-to-cell communication. Kidney Int 2010, 78:838-848.

27. Shabbir A, Cox A, Rodriguez-Menocal L, Salgado M, Badiavas EV: Mesenchymal Stem Cell Exosomes Induce Proliferation and Migration of Normal and Chronic Wound Fibroblasts, and Enhance Angiogenesis In Vitro. Stem Cells Dev 2015, 24:1635-1647.

28. Zhang B, Wang M, Gong A, Zhang X, Wu X, Zhu Y, Shi H, Wu L, Zhu W, Qian $\mathrm{H}, \mathrm{Xu} \mathrm{W}$ : HucMSC-Exosome Mediated-Wnt4 Signaling Is Required for Cutaneous Wound Healing. Stem Cells 2015, 33:2158-2168.

29. Sharma GD, He J, Bazan HE: p38 and ERK1/2 coordinate cellular migration and proliferation in epithelial wound healing: evidence of cross-talk activation between MAP kinase cascades. J Biol Chem 2003, 278.21989-21997.

30. Satoh Y, Saitoh D, Takeuchi A, Ojima K, Kouzu K, Kawakami S, Ito M, Ishihara M, Sato S, Takishima K: ERK2 dependent signaling contributes to wound healing after a partial-thickness burn. Biochem Biophys Res Commun 2009, 381:118-122.

31. Cheng B, Liu HW, Fu XB, Sun TZ, Sheng ZY: Recombinant human platelet-derived growth factor enhanced dermal wound healing by a pathway involving ERK and c-fos in diabetic rats. J Dermatol Sci 2007, 45:193-201.

32. Pintucci G, Moscatelli D, Saponara F, Biernacki PR, Baumann FG, Bizekis C, Galloway AC, Basilico C, Mignatti P: Lack of ERK activation and cell migration in FGF-2-deficient endothelial cells. FASEB J 2002, 16:598-600.

33. Herrmann JL, Weil BR, Abarbanell AM, Wang Y, Poynter JA, Manukyan MC, Meldrum DR: IL-6 and TGF-alpha costimulate mesenchymal stem cell vascular endothelial growth factor production by ERK-, JNK-, and PI3K-mediated mechanisms. Shock 2011, 35:512-516.

34. Medina RJ, O'Neill CL, O'Doherty TM, Knott H, Guduric-Fuchs J, Gardiner TA, Stitt AW: Myeloid angiogenic cells act as alternative M2 macrophages and modulate angiogenesis through interleukin-8. Mol Med 2011, 17:1045-1055.

35. Jorda M, Vinyals A, Marazuela A, Cubillo E, Olmeda D, Valero E, Cano A, Fabra A: Id-1 is induced in MDCK epithelial cells by activated Erk/MAPK pathway in response to expression of the Snail and E47 transcription factors. Exp Cell Res 2007, 313:2389-2403.

36. Li T, Hu J, Du S, Chen Y, Wang S, Wu Q: ERK1/2/COX-2/PGE2 signaling pathway mediates GPR91-dependent VEGF release in streptozotocin-induced diabetes. Mol Vis 2014, 20:1109-1121.

37. Wu G, Luo J, Rana JS, Laham R, Sellke FW, Li J: Involvement of COX-2 in VEGF-induced angiogenesis via P38 and JNK pathways in vascular endothelial cells. Cardiovasc Res 2006, 69:512-519.

38. Berra E, Pages G, Pouyssegur J: MAP kinases and hypoxia in the control of VEGF expression. Cancer Metastasis Rev 2000, 19:139-145.

39. Montecalvo A, Larregina AT, Shufesky WJ, Stolz DB, Sullivan ML, Karlsson JM, Baty CJ, Gibson GA, Erdos G, Wang Z, et al: Mechanism of transfer of functional microRNAs between mouse dendritic cells via exosomes. Blood 2012, 119:756-766.

40. Hergenreider E, Heydt $\mathrm{S}$, Treguer $\mathrm{K}$, Boettger $\mathrm{T}$, Horrevoets AJ, Zeiher AM, Scheffer MP, Frangakis AS, Yin X, Mayr M, et al: Atheroprotective communication between endothelial cells and smooth muscle cells through miRNAs. Nat Cell Biol 2012, 14:249-256.

41. Ramachandran S, Palanisamy V: Horizontal transfer of RNAs: exosomes as mediators of intercellular communication. Wiley Interdiscip Rev RNA 2012, 3:286-293.
42. Valadi $\mathrm{H}$, Ekstrom $\mathrm{K}$, Bossios $\mathrm{A}$, Sjostrand $\mathrm{M}$, Lee JJ, Lotvall JO: Exosome-mediated transfer of mRNAs and microRNAs is a novel mechanism of genetic exchange between cells. Nat Cell Biol 2007, 9:654-659.

43. Mittelbrunn M, Gutierrez-Vazquez C, Villarroya-Beltri C, Gonzalez S, Sanchez-Cabo F, Gonzalez MA, Bernad A, Sanchez-Madrid F: Unidirectional transfer of microRNA-loaded exosomes from $\mathrm{T}$ cells to antigen-presenting cells. Nat Commun 2011, 2:282

44. Lv LL, Cao Y, Liu D, Xu M, Liu H, Tang RN, Ma KL, Liu BC: Isolation and quantification of microRNAs from urinary exosomes/microvesicles for biomarker discovery. Int J Biol Sci 2013, 9:1021-1031.

45. Zhou Q, Li M, Wang X, Li Q, Wang T, Zhu Q, Zhou X, Wang X, Gao X, Li X: Immune-related microRNAs are abundant in breast milk exosomes. Int J Biol Sci 2012, 8:118-123.

46. Wang X, Gu H, Oin D, Yang L, Huang W, Essandoh K, Wang Y, Caldwell CC, Peng T, Zingarelli B, Fan GC: Exosomal miR-223 Contributes to Mesenchymal Stem Cell-Elicited Cardioprotection in Polymicrobial Sepsis. Sci Rep 2015, 5:13721.

47. Cui Y, Luan J, Li H, Zhou X, Han J: Exosomes derived from mineralizing osteoblasts promote ST2 cell osteogenic differentiation by alteration of microRNA expression. FEBS Lett 2016, 590:185-192.

48. Liu LZ, Li C, Chen $\mathrm{O}$, Jing Y, Carpenter R, Jiang Y, Kung HF, Lai L, Jiang BH: MiR-21 induced angiogenesis through AKT and ERK activation and HIF-1alpha expression. PLoS One 2011, 6:e19139.

49. Goodwin AJ Guo C, Cook JA, Wolf B, Halushka PV, Fan H. Plasma levels of microRNA are altered with the development of shock in human sepsis: an observational study. Crit Care 2015, 19:440. 